\title{
Einleitung: Arbeit und gesellschaftlicher Wandel
}

\author{
Brigitte Aulenbacher $\cdot$ Meinrad Ziegler
}

Die soziologische Arbeits- und Geschlechterforschung und ihr verbundene Gesellschaftsanalysen tragen seit geraumer Zeit wesentlich dazu bei, den gesellschaftlichen Wandel, der sich seit Mitte der 1970er Jahre in allen OECD-Ländern vollzieht, zeitgeschichtlich einzuordnen und seine Ursachen, seine Dynamiken, seinen Verlauf und seine Auswirkungen zu begreifen. Weitgehend konsensuell werden die folgenden Diagnosen vorgetragen:

National wie international bedeutsame zeitgeschichtliche Zäsuren sind im Zusammenbruch der staatssozialistischen Gesellschaften Osteuropas und in der Globalisierung zu sehen. Die mit der Ära des Fordismus verbundene geregelte Ordnung von wechselseitigen Rechten und Verpflichtungen zwischen Arbeit und Kapital weicht zusehends kurzfristigen Arrangements, was die Deregulierung und Flexibilisierung des Normalarbeitsverhältnisses zur Folge hat (vgl. Sennett 1998; Bauman 2004). Die Lebensform der Kleinfamilie nach dem Ernährer- und Hausfrauenmodell hat ihre Bedeutung als normatives Ideal eingebüßt. In Verbindung mit Bildungsreformen und der formalrechtlichen Gleichstellung der Geschlechter sind heute vielfältige Lebensformen weitgehend akzeptiert; allerdings lassen sich im Hinblick auf die praktizierten Partnerschaftsmodelle doch milieuspezifische Differenzierungen beobachten (vgl. Kohlmorgen 2004; Ziegler 2005). Die seit den 1990er Jahren erfolgte Öffnung des globalen Wirtschaftsraumes ermöglichte es der Finanzwirtschaft, sich von der Realökonomie zu entkoppeln und Reorganisationen in den Strukturen der Produktion und des Managements zu forcieren, die die Arbeits- und Lebensverhältnisse der Menschen im Vergleich zu ihrer vorherigen fordistischen Absicherung tiefgreifend entsichert haben (vgl. Dörre/Brinkmann 2005). Diese Entwicklungen gingen außerdem mit Migrationsbewegungen historisch neuen Ausmaßes einher (vgl. Morokvasic-Muller 2003).

Erfordernisse gesellschaftlicher Reproduktion, deren Bearbeitung unter fordistischen Vorzeichen stabil in und zwischen Markt, Staat, Drittem Sektor und Privathaushalt austariert war, werden unter vorrangig ökonomischen Aspekten seither neu gewichtet. Damit verbunden wird die zu ihrer Gewährleistung notwendige Arbeit in und zwischen diesen ihrerseits ebenfalls erheblich gewandelten Institutionen und zugleich nach Geschlecht, Ethnie, Schicht neu verteilt (vgl. Aulenbacher/Riegraf 2009; Young 1998). Durch all diese Entwicklungen hindurch lassen sich national wie international Verschiebungen im Verhältnis von Markt und Demokratie verzeichnen, in deren Folge das ökonomische Geschehen seit den 1990er Jahren die gesellschaftliche Entwicklung unkontrollierter, unregulierter und unbeeinflussbarer als zuvor prägt (vgl. Aulenbacher 2009; Negt 2001; Young 2006). 
Mit dem Thema Arbeit in Alltag, Biografie, Gesellschaft bewegt sich das vorliegende Heft der Österreichischen Zeitschrift für Soziologie im größeren Rahmen der hier skizzierten soziologischen Sozial- und Zeitdiagnostik. Wir versuchen allerdings, innerhalb dieser Forschungsstränge einige Akzente im Hinblick auf weitgehende Leerstellen und Ausblendungen zu setzen, die in zahlreichen selbstkritischen Debatten der vergangenen Jahre moniert wurden.

Eine der weitgehend unbearbeiteten Perspektiven ist im Anschluss an kritische Interventionen, welche die Soziologie zu einer grundlagentheoretisch erneuerten Revision der europäischen Moderne auffordern (vgl. Knapp 2008), darin auszumachen, dass die Fragestellungen und Schwerpunkte im Feld der Arbeitsforschung nicht frei sind von eurozentrischen Akzentsetzungen. Zwar sind die angesprochenen Dynamiken, die aus den ökonomischen Neuformierungen, politischen Regulativen und Formen der Sozialorganisation der Länder des Westens hervorgehen, von Bedeutung über sie hinaus und werden auch mit Entwicklungen in Beziehung gesetzt, welche aus anderen Regionen der Welt hervorgehen. Letztere werden aber kaum unmittelbar in den Blick genommen.

Eine zweites unterbelichtetes Feld sind mikrosoziologische Dimensionen der angesprochenen Prozesse des sozialen Wandels. Wenn es um die Frage nach den Dynamiken sozialen Wandels geht, sind traditionell strukturtheoretische und makrosoziologische Perspektiven vorherrschend. Ein differenziertes Verständnis der aktuellen Transformationen in den eingelebten Arrangements zwischen Arbeit und Kapital, zwischen Markt und Formen der Staatlichkeit stellt sich ein, wenn der Blick auch darauf gerichtet wird, wie globale Prozesse und Veränderungen auf alltägliche Lebenswelten einwirken und welches Handeln sich dort in Auseinandersetzung mit neuen strukturellen Rahmungen herausbildet. Gerade in Perioden großer Transformationen greift eine ausschließlich auf Strukturdaten konzentrierte Soziologie zu kurz, weil diese vor allem das Regelmäßige und Sich-Wiederholende erfasst, nicht aber die Entstehung jener neuen Strategien des Handelns und biografischer Orientierungen, mit denen soziale Gruppen und einzelne AkteurInnen auf veränderte Anforderungen antworten (vgl. Ziegler 2000).

Eine dritte Fragestellung, die es im Zusammenhang mit der aktuellen Dynamik des sozialen Wandels zu thematisieren lohnt, ist jene nach den demokratiepolitischen Implikationen der sozialen Konflikte, die in den skizzierten Neuordnungen der Arbeits- und Lebensverhältnisse eingeschrieben sind. Neben dem in der soziologischen Sozialdiagnostik in erster Linie betrachteten Widerspruch kapitalistischer Industriegesellschaften - jenem zwischen Arbeit und Kapital - verändert sich auch jener zwischen den Räumen der Arbeitswelt und dem Privaten, zwischen den Bedürfnissen der Produktion und jenen der Reproduktion (vgl. Becker-Schmidt/Krüger 2009). Dieses Konfliktfeld gefährdet nicht nur unmittelbar die Bedingungen der individuellen Reproduktion, also den Erhalt von Identität, Körperlichkeit und sozialen Bindungen, sondern berührt auch die Reproduktion des Gemeinwesens und die Bedingung der Möglichkeit unterschiedlicher normativer Modelle von Demokratie (vgl. Habermas 1996; Negt 2001). Die gesellschaftliche Durchsetzung von Arbeits- und Lebensbedingungen, die es den Mitgliedern einer Gesellschaft nicht mehr möglich machen, den privaten Raum der Produktion und Reproduktion zu überschreiten, sind kompatibel mit dem Modell der liberalen Demokratie, geraten jedoch notwendig in Konflikt mit den Modellen einer republikanischen oder deliberativen Demokratie, in denen Politik mehr als nur die Moderation und Durchsetzung 
von privaten Interessen gegenüber einem als Verwaltungsapparat gedachten Staat bedeutet. Das Themenheft führt verschiedene Perspektiven auf den gesellschaftlichen Wandel zusammen. Mit dem besonderen Gewicht auf seine alltägliche und biografische Bearbeitung und einem Blick auf globale Entwicklungen werden einige jener Aspekte aufgegriffen, die angesichts der genannten Beschränkungen in sozial- und zeitdiagnostischen Betrachtungen eher am Rande stehen.

Joe L. P. Lugalla und Huruma L. Sigalla zeigen anhand einer ethnografischen Regionalstudie aus Tanzania, wie sich Kinderarbeit in Abhängigkeit der Ausbreitung von Aids entwickelt. HalbwaisInnen und WaisInnen, deren Eltern an Aids verstorben sind, arbeiten unter menschenunwürdigen Bedingungen in Haushalten und Betrieben der regionalen Nahrungsmittelindustrie, um ihr und teilweise auch das Überleben weiterer Familienangehöriger zu sichern. Diese Form der Arbeit (childlabour) entzieht sich zum einen politischen und gesetzlichen Regulierungen und wird zum anderen dadurch verdeckt gehalten, dass sie in einer Gesellschaft stattfindet, in der Kinderarbeit (childwork) ein traditioneller Bestandteil der Integration in die Gemeinschaft ist.

Die stabile Trennung von produktiver und unproduktiver Arbeit, wobei letztere in Form der Hausarbeit zugleich gesellschaftlich weitgehend unsichtbar ist, beschäftigt Helma Lutz. Sie zeigt am Beispiel Deutschlands, wie Arbeit entlang dieser Trennung von der Kolonialzeit bis heute nach Geschlecht und ,Rasse' verteilt worden ist. In der gegenwärtigen Adult-Worker-Gesellschaft werde dabei in mehr oder minder regulierter Form und verbunden mit der Herausbildung ganz neuer Beschäftigungs- und Lebensformen insbesondere auf die Arbeitskraft von Migrantinnen zugegriffen, welche nach den osteuropäischen Grenzöffnungen in neuem Ausmaß verfügbar geworden ist.

Wenngleich der Zusammenbruch des Staatssozialismus international eine Zäsur markiert, so ist die Situation in Ostdeutschland doch besonders. Dort schlägt sich die gesellschaftliche Transformation bei denjenigen, die sie erlebt haben, als biografischer Bruch nieder und außerdem als Bruch zwischen ihnen und der nachfolgenden Generation. Susanne Völker zeigt anhand der Fallgeschichte einer prekär beschäftigten Einzelhandelsverkäuferin, die sie in einer praxeologischen Betrachtungsweise vorstellt, wie gesellschaftliche Verunsicherungen alltäglich und biografisch bearbeitet und dabei auch generationenbezogene Unterschiede deutlich werden.

Raewyn Connells Untersuchung führt in das mittlere und obere Management der australischen Finanzökonomie. Mit zwei biografischen Fallstudien zeigt sie, wie sich unterschiedliche Formen von Männlichkeit aus sozialisatorischen Vorprägungen und individuellen Lebensperspektiven auf der einen Seite und institutionellen Anforderungen der Unternehmen auf der anderen Seite konstituieren und welche Friktionen zwischen beiden Dimensionen einer Lebensgeschichte auftauchen können. In der Darstellung biografischer Verläufe wird deutlich, wie und in welchem Ausmaß global agierende Unternehmen in der Lage sind, sich lebendige Arbeit anzueignen und damit auch bestimmte Geschlechterregimes zu implementieren. Zugleich werden an jedem Fall auch spezifische soziale oder physische Grenzen sichtbar, die auf das Moment der Unverfügbarkeit in diesen Prozessen verweisen.

Hubert Eichmann nähert sich der Wiener Kreativwirtschaft anders, als es in Forschungen zu diesem Sektor üblicherweise der Fall ist. Statt von den Besonderheiten der Beschäftigungsverhältnisse auszugehen, setzt er bei den beruflichen Orientierungen der dort Be- 
schäftigten an. Der Vorteil dieses Zugangs besteht darin, dass die jeweiligen beruflichen Selbstkonzepte gewissermaßen als subjektive Taktgeber für die konkreten Arbeitspraktiken und ihre Wahrnehmung fungieren. Der Beitrag veranschaulicht die Sinnhaftigkeit der entwickelten Typologie von beruflichen Orientierungen und geht vertiefend auf den Typus des „Kunst-Schaffens“ ein, der sich durch subjektive Konzepte einer Verschmelzung von Arbeit und Leben im Sinn eines ganzheitlichen Lebensentwurfs auszeichnet.

Wenn in Bezug auf die westeuropäische Entwicklung ein bedeutender zeitgeschichtlicher Einschnitt an der Erosion des Normalarbeitsverhältnisses festgemacht wird, so darf nicht verkannt werden, dass diese Norm und Institution nie gleichermaßen für alle Erwerbstätigen galt, sondern in erster Linie und im vollen Umfang der damit verbundenen Privilegien für die einheimische männliche Mittelschicht. Mit der Zersetzung dieses Arrangements ist, so zeigt die Analyse von Diana Lengersdorf und Michael Meuser, daher ein spezifischer Konnex von Arbeit und Männlichkeit ins Wanken geraten. Die identitätsstiftende Bedeutung, die Erwerbsarbeit für Männer als Männer hat, wird davon zwar auch berührt, aber letztlich nicht in Frage gestellt. Es bilden sich im Wandel von Arbeit auch neue Formen von Männlichkeit heraus.

Der Beitrag von Edgar Forster wirft die Frage auf, wie sich die neuen neoliberalen Konzepte über Arbeit mit den normativen Idealen der bürgerlichen Gesellschaft über politische Öffentlichkeit verbinden oder vereinbaren lassen. Der Autor diskutiert vorerst kritisch unterschiedliche theoretische Erklärungen für das Verschwinden des Politischen, wie es in vielen sozialwissenschaftlichen Diagnosen betont wird: Westliche Gesellschaften würden nicht vom demos, sondern von Eliten und transnationalen Konzernen regiert. Das zentrale Argument besteht dann darin, einen Zusammenhang zwischen Erosionserscheinungen liberaler Demokratien und dem diskursiven Konzept des Humankapitals herzustellen. In einer gesellschaftlichen Ordnung, die Arbeit in Humankapital transformiere - und sich insofern einer Unterordnung aller reproduktiven Tätigkeiten unter die Steigerung der individuellen Arbeitsfähigkeit verschreibe -, sei Politik nur mehr als Abfolge von institutionellen Verwaltungsakten denkbar, in der Prozesse der demokratischen Meinungs- und Willensbildung zu Fragen des öffentlichen Interesses keinen sozialen Ort mehr haben würden.

\section{Literatur}

Aulenbacher, Brigitte. 2009. Arbeit, Geschlecht und soziale Ungleichheiten, Perspektiven auf die Krise der Reproduktion und den Wandel von Herrschaft in der postfordistischen Arbeitsgesellschaft. In: Arbeits- und Industriesoziologische Studien (AIS) der Sektion Arbeits- und Industriesoziologie in der DGS 2: 61-78. www.ais-studien.de.

Aulenbacher, Brigitte und Birgit Riegraf. 2009. Markteffizienz und Ungleichheit - Zwei Seiten einer Medaille? Klasse/Schicht, Geschlecht und Ethnie im Übergang zur postfordistischen Arbeitsgesellschaft. In: Arbeit, Diagnosen und Perspektiven der Geschlechterforschung, Forum Frauen- und Geschlechterforschung Band 25, hrsg. Brigitte Aulenbacher und Angelika Wetterer, 230-248. Münster: Westfälisches Dampfboot.

Becker-Schmidt, Regina und Helga Krüger. 2009. Krisenherde in gegenwärtigen Sozialgefügen: Asymmetrische Arbeits- und Geschlechterverhältnisse - vernachlässigte Sphären gesellschaftlicher Reproduktion. In: Arbeit. Perspektiven und Diagnosen der Geschlechterforschung, hrsg. Brigitte Aulenbacher und Angelika Wetterer, 12-41. Münster: Westfälisches Dampfboot. 
Dörre, Klaus und Ulrich Brinkmann. 2005. Finanzmarktkapitalismus. Triebkraft eines flexiblen Produktionsmodells? In: Finanzmarktkapitalismus. Analysen zum Wandel von Produktionsregimen, hrsg. Paul Windorf, 85-116. Wiesbaden: VS Verlag für Sozialwissenschaften.

Bauman, Zygmunt. 2004. Aufstieg und Niedergang der Arbeit. In: Die Gesellschaft im 21. Jahrhundert. Perspektiven auf Arbeit, Leben, Politik, hrsg. Gerhard Gamm, Andreas Hetzel und Markus Lilienthal, 23-37. Frankfurt/M. und New York: Campus.

Habermas, Jürgen. 1996. Drei normative Modelle von Demokratie. In: Ders. Die Einbeziehung der Anderen. Studien zur politischen Theorie, 277-292. Frankfurt/M.: Suhrkamp.

Knapp, Gudrun-Axeli. 2008. Verhältnisbestimmungen: Geschlecht, Klasse, Ethnizität in gesellschaftstheoretischer Perspektive. In: ÜberKreuzungen, Fremdheit, Ungleichheit, Differenz, hrsg. Cornelia Klinger und Gudrun-Axeli Knapp, 138-170. Münster: Westfälisches Dampfboot.

Kohlmorgen, Lars. 2004. Regulation, Klasse, Geschlecht, Die Konstituierung der Sozialstruktur im Fordismus und Postfordismus. Münster: Westfälisches Dampfboot.

Morokvasic-Muller, Mirjana. 2003. Gender-Dimensionen der postkommunistischen Migrationen in Europa. In: Migration, Biographie und Geschlechterverhältnisse, hrsg. Ursula Apitzsch und Mechthild M. Jansen, 143-171. Münster: Westfälisches Dampfboot.

Negt, Oskar. 2001. Arbeit und menschliche Würde. Göttingen: Steidl Verlag.

Sennett, Richard. 1998. Der flexible Mensch. Die Kultur des neuen Kapitalismus. Berlin: Berlin Verlag.

Young, Brigitte. 1998. Genderregime und Staat in der globalen Netzwerk-Ökonomie. In: Prokla 111, 28: 175-198.

Young, Brigitte. 2006. Geschlechter(un)gleichheit und Öffnung der globalen Märkte. In: Die Neuverhandlung sozialer Gerechtigkeit, Feministische Analysen und Perspektiven, hrsg. Ursula Degener und Beate Rosenzweig, 151-164. Wiesbaden: VS Verlag für Sozialwissenschaften.

Ziegler, Meinrad. 2000. Das soziale Erbe. Eine soziologische Fallstudie über drei Generationen einer Familie. Wien, Köln und Weimar: Böhlau.

Ziegler, Meinrad. 2005. Vater, Mutter, Kind: Familienbilder und Familienwirklichkeiten. In: Heimat bist du großer Söhne. Gaismair-Jahrbuch 2005, hrsg. Alexandra Weiss u. a., 173-188. Innsbruck: StudienVerlag.

Brigitte Aulenbacher, Professorin für Soziologische Theorie und Sozialanalysen (unter besonderer Berücksichtigung der Gender-Dimensionen) am Institut für Soziologie der Johannes-Kepler-Universität Linz. Arbeitsgebiete sind: Gesellschaftstheorie, Methodologie, Geschlechter-, Rationalisierungs-, Arbeits- und Organisationsforschung. Aktuelle Publikationen: Arbeit, Perspektiven und Diagnosen der Geschlechterforschung, Münster 2009 (hrsg. mit Angelika Wetterer); Erkenntnis und Methode, Geschlechterforschung in Zeiten des Umbruchs, Wiesbaden 2009 (hrsg. mit Birgit Riegraf). Adresse: Johannes-Kepler-Universität, Institut für Soziologie, Altenberger Str. 69, A-4040 Linz. E-Mail: brigitte.aulenbacher@jku.at.

Meinrad Ziegler, a. Univ.-Prof. am Institut für Soziologe der Johannes Kepler Universität Linz. Arbeitsschwerpunkte: Soziologische Theorie, Methodologie und Methoden der qualitativen Sozialforschung, Biographieforschung. Aktuelle Publikationen: Heteronormativität und Homosexualitäten, Innsbruck/Wien/Bozen 2008 (als Herausgeber gem. mit Rainer Bartel/Ilona Horwath/ Waltraud Kannonier-Finster/Maria Mesner/Erik Pfefferkorn); Wahrheit, Sprache, Rhetorik. Der schwierige Anspruch auf wahres Wissen in den Sozialwissenschaften. In: Brigitte Aulenbacher/ Meinrad Ziegler (Hrsg.): In Wahrheit ... Herstellung, Nutzen und Gebrauch von Wahrheit in Wissenschaft und Alltag. Innsbruck/Wien/Bozen 2010. Adresse: Johannes-Kepler-Universität, Institut für Soziologie, Altenberger Str. 69, A-4040 Linz. E-Mail: meinrad.ziegler@jku.at. 